\title{
Teaching Principle and Instrutional Design Analyze in Linear Algebra Course Based on Problem-based Learning
}

\author{
Liang Yan, Wu Simin* \\ School of Science, Guangdong University of Petrochemical Technology, Guangdong, China
}

Keywords: Problem-based Learning, Linear Algebra, Analysis of Instructional Design, Determinant, Matrix

Abstract: Linear algebra is an important compulsory course for undergraduate students majoring in science and engineering, as well as some economic and management students. However,the teaching effect is not satisfiedin the current teaching and learning.In order to improve the teaching effect, this paper researches the basic teaching principles of problem-based learning in linear algebra course based on the analysis of the current teaching and learning. It finds out that because the highly abstract of this course, the traditional teaching pattern is not suitable for linear algebra curse, while problem-based learning can achieve better result. What's more, it also provides several examples of instructional design using problem-based learning on the main concepts of linear algebra, such as determinant, matrix and vector space. These examples discuss how to design the problems to help the students for understanding the abstract concepts and construct a stable and precise concept map.The analysis and the examples are meaningful for both teaching theory and practice.

\section{Introduction}

With therapid development ofcomputer science and big data processing technology, linear algebra is becoming increasingly important in university and college curriculum system. According to the current teaching requirement of universities, Linear algebra is a compulsory course for undergraduate students majoring in science and engineering as well as some economic and management students ${ }^{[1]}$.In the past decades, it is offering more and more widely in various majors. This course is the foundation for students to learn other specialized courses, as well as provides necessary training for students' innovative consciousness and the way of thinking. It is a powerful tool for students to solve specialized problems, which plays an extremely vital role in students' curriculum system, and affects the depth and potential of students' knowledge development. Las Garding, a Swedish mathematician, proposed in his book"Encounter with Mathematics" that "it will be looks like a illiteracy if you are not familiar with the concept of linear algebra and go to study natural science" ${ }^{\text {"2] }}$. Therefore, the status and importance of the linear algebra course is self-evident. 


\section{The Current Teaching and Learning of Linear Algebra Teaching}

Linear algebra course is so important, but in the current practical teaching, we found that its teaching effect is not satisfied. Compared with other courses, there are a lot of definitions, theorems and symbolized proofs in linear algebra textbook. These definitionsare concise and precise expression obtained after a long exploration, thinking, and summary of many generations of mathematicians, which areelaborated in a highly abstract axiomatic language.These expressionsare a bigprogress in mathematics, but they omit the fiery and interesting thinking process of mathematicians. As a result, students feel very abstract and difficult to understand them.In addition, the concepts in linear algebra are all defined in a high-dimensional vector space, while the real world that students live in is only three-dimensional. This also brings difficulties for students to understand. Difficulties in understanding the concepts cause the many students' fear of learning linear algebra. They feel it has too many boring algorithms and abstract profound definitions, which leads to losttheir interest in learning.Consequently, they are easy to fall into a bad learning mode,which is crazy memorizing algorithms before the exam, and forgetting them all after the exam.

According to the discussion above, it can be found that the understanding of concepts in linear algebra teaching has a crucial impact on students' learning. If students don't understand the concept deeply, they will be unable to understand the theorems, proofs and algorithms. Then, they will definitely lose their interest in learning. There are two main reasonsthis phenomenon.On one hand, most colleges and universities still adopt the traditional teaching mode, which follow the teaching process of "Concept-Theorem-Exercise" inteaching linear algebra ${ }^{[3]}$. Most teachers pay more attention to explain the problem-solving skills in the class, butfail to make a good explanation of the origin of definitions and theorems, the application backgrounds, as well as the mathematical thinking method. Thus, students can't understand the how the definitions and theorems comes out and what they are application. As a result, they can only memorize them passively and hard to achieve good teaching effect. On the other hand, the current teaching process in linear algebra is to teach concepts one by one separately without explain the connection between different concepts. Students are unable to find the relationships between various concepts and theorems independently. Then they will be failed to discover mathematical nature and build a perfect knowledge network.This will lead to the fact that students still can't flexibly apply the knowledge they have learned in solving problems.

\section{The Theoretical Analysis of Problem-Based Learning in Linear Algebra Teaching}

Problem-Based Learningis a learner-centered, problem driventeaching method,which makes use of the suitable research problems based on the learners' existing knowledge foundation ${ }^{[4]}$. It aims to enable learners to conduct research, integrate theory and practice, and put forward feasible solutions to a given research problems. The great educator Confucius said,"One should not enlighten a student until he tries to understand but can't get it, and should not give guidance to a student until he understands in his heart but can't express it perfectly". This kind of teaching methodenables students "learn by doing", which can make students change from traditional passive acceptance learning pattern to active thinking and internalizationlearning method.Psychologist Piagetbelieves that knowledge develops in a way of answering questions ${ }^{[5,6]}$. He proposed the famous constructivist epistemology, whichprovides psychological theoretical foundation forProblem-based Learning teaching model.Multiple word-wideteaching empirical researchesshow thatProblem-based Learningcan effectively promote learners' research ability, innovation ability and the ability for problem solving $^{[6-8]}$. The difficulty of teaching and learninglinear algebra lies in the understanding 
of abstract concepts and symbolic theorems. Problem-based Learning can solve this difficult efficiently. By using appropriate problem, it makes students form a cognitive gap.Thenin the process of active thinking, discussion and problemsolving, students can realize the process of generating concepts and theorems, build new concepts and theorems connected with existing knowledge, and integrate them into their existing knowledge network. The experience of active research instead of passive acceptance and rote memorization of abstract concepts, students will get more profound understanding and their interest in learning and sense of achievement will also be improved.

Problem-based Learningprovides a good theory and teaching model for teaching abstract concepts and symbolic theorems in linear algebra. The key to implementation this teaching model is how todesignproblems which aresuitable, effective and can stimulating students' interest in learning.According to the teaching principles of Problem-based learning, the designed problems should meet the following characteristics. 1) Can be connected with students' existing knowledge; 2) Can form students' cognitive gaps and stimulate students' interest in learning; 3) Bysolving the problems can help students to construct new concepts and integrate them into the existing knowledge system. It can be seen that the quality of problems will directly affect the teaching effect ofproblem based learning.

\section{Examples and Analysis of Problem-Based Learning in Linear Algebra Teaching}

The current linear algebra course usually introduces determinant, matrix, vector, linear equations, vector space, etc. In this section, we selected some contents of linear algebra course, based on the practical teachingfor engineeringundergraduate students with general mathematical foundation,to provide some examples of problems designed using Problem-based Learning method. In addition, we also give out the analysis of the examples.

\subsection{Example of Problems on Teaching Determinant}

The concept and theory of determinant was formed in the 17th century to the 19th century. Cramer, a mathematician in Athens,create and used determinant to give a simple algorithm for solvingthe first-order equations, as well as a method to judge whether it is a solution (Cramer's rule). Therefore, the determinant originates from the solution of solving linear equations.For undergraduate students, using elimination method to solve linear equations is amain content in middle school, which students are very familiar with. Thus, the way to obtain the concept of determinantby exploring the problem of solving linear equations can make students more acceptable in emotional attitude, and also easy to stimulate students' interest in learning.

Problem1.1 Please use elimination method to solve the followingtwo-variables linear equations

$$
\left\{\begin{array}{l}
a_{11} x_{1}+a_{12} x_{2}=b_{1} \\
a_{21} x_{1}+a_{22} x_{2}=b_{2}
\end{array}\right.
$$

In high school, most of the linear equations solved by students are linear equations withspecific coefficients and right hand-side. In this case, although the coefficients and the righthand-side are represented by symbols, most students can still get the general solution of the equation system by the elimination method, because the number of unknownsin this equations system is small. The general solution of this system is 


$$
\left\{\begin{array}{l}
x_{1}=\frac{b_{1} a_{22}-a_{12} b_{2}}{a_{11} a_{22}-a_{12} a_{21}} \\
x_{2}=\frac{a_{11} b_{2}-b_{1} a_{21}}{a_{11} a_{22}-a_{12} a_{21}}
\end{array}\right.
$$

Problem1.2 Does this general form of solution always exists?

When students gain the general form of the solution in the first problem, they will have a sense of achievement. They may think that they have solved the Problem 1.1 perfectly. Thus, the Problem 1.2 is unexpected to some students which will cause their attention and interest for thinking. According to practical teaching, it's not hard for a careful student to find that the denominator of the solution formula must be non-zero to make sure the formula is meaningful. And the denominator is exactly a second-order determinant. This provide the foundation for students to learn the concept of determinant and Clem's rule.

Problem1.3 We have already known that the system of equations in Problem 1.1 has a unique solution if and only ifthe denominator is non-zero.Thus, the expression of the denominator is important. Is there any way to remember it quickly?

Teachers can introduce the note of second order determinant by this problem. The expression of the denominator can be represented by second order determinant

$$
\left|\begin{array}{ll}
a_{11} & a_{12} \\
a_{21} & a_{22}
\end{array}\right|
$$

The elements in this note is correspond with the coefficients of the original linear system. The calculate algorithm is the product of the elements in the main diagonal subtract the product of the elements in the counter-diagonal. It is easy for remember and concise. As a result, students can construct the concept of second order determinant clearly.

Problem1.4 Can the second order determinant be generalized to thirdorder determinant?

The second order determinant is gained by solving two-variables linear equations. Therefore, teacher can guide student to find out third-order determinant should be gained from solving three -variable linear system by analogy. It is easy for students to get the note of three order determinant is

$$
\left|\begin{array}{lll}
a_{11} & a_{12} & a_{13} \\
a_{21} & a_{22} & a_{23} \\
a_{31} & a_{32} & a_{33}
\end{array}\right|
$$

The formula for it is the denominator of the general solution in tree-variables linear system. From this way, students can have a deeper understanding of the conciseness of determinant notation and better acceptance of new concepts.

Problem1.5 Can the concept of the thirdorder determinant continue be generalized to fourth, fifth and nthorder?

This problem promotes students continue research generalizing from the specialized second, third order to general nth order. By analogy, students can get the note of nth order determinant easily. However, it is hard for them to find out the formula of nth order determinant. From special to general is a common way of thinking in mathematics research.And inductive summary is a common method to get results from special to general. Based on this, teacher can continue to guide by the 


\section{following problem.}

Problem1.6 Is there any common law in the formula of second and third order determinants?

This problem enables students to return to the existing knowledge, which contribute to form good thinking habits. In solving this problem, students can revise and analyze the formula of second and third order determinants, summarized the common laws under teacher's guidance, generalized the concept of determinant.

Problem1.7 Can you give a definition of nthorder determinant?

Though the summery before, students can find that 1) there are $n$ ! items in the formula of anth order determinant; 2) each item has n elements; 3) the elements in each item come from different rows and columns in the determinant; 4) the sign of each item is determined by the sum of the reverse order numbers of the elements' column arrangement. Based on these common laws, students translate them into mathematical symbols and get the definition of the determinant

$$
D_{n}=\sum(-1)^{N\left(m_{1} m_{2} \cdots m_{n}\right)} a_{1 m_{1}} a_{2 m_{2}} \cdots a_{n m_{n}}
$$

The above seven questions are go forwardone by one in the teaching concept of determinant, and guide students to reproduce and experience the thinking process of the generalization of determinant. Each problem is a "scaffold" for students to explore the next problem, which helps students realize the transition from known to new knowledges. This process of acquiring new knowledges is related to the students' existing knowledge structure, which makes them easier to absorb and internalize the new knowledges and understand the new concept more accurately.In this way, the learning of definition of determinant is not through the cold and boring mathematical expression, but the result of students' own exploration and induction, which is more impressive.

\subsection{Example of Problems on Teaching Matrix Inverse}

Matrix is a main research object and the most important tool for solving problems in linear algebra. It has a very wide application in many fields ${ }^{[9]}$. Matrixinverse is an important concept in matrix theory and a crucial basis for understanding and applying other properties of matrix

Problem2.1 An arbitrary non-zero real number $a$, there exists another non-zero real number $a^{-1}$, such that

$$
a a^{-1}=a^{-1} a=1
$$

Analogy to matrix, for an arbitrary non-zero matrix $A$, is there exists another matrix $B$, satisfy

$$
A B=B A=E
$$

This problem is designed by analogy, generalized from the property of real number to matrix.Therefore, students can get the definition of matrixinverse from their familiar knowledges.

Problem2.2 What problems do you think are worth studying about matrix inverse we have just learned?

This problem can guide students to find and put forward problems, and then study them.Learning to ask questions contributes to cultivate students' ability of innovation, and research. The following are the problems for matrix inverse.

Problem2.3 If a matrix has an inverse, is the inverse unique?

Problem2.4 Does every matrix have an inverse? Does every non-zero matrix have an inverse? Let non-zero matrix

$$
A=\left(\begin{array}{ll}
1 & 0 \\
0 & 0
\end{array}\right)
$$

can you find the inverse of it?

Problem2.5 What is the necessary and sufficient condition of invertible matrix? 
Problem2.6 How to find the inverse of ainvertible matrix?

Problem2.7 Based on the determinant and matrix operation, what properties of the invertible matrix can you discover?

Actually, these problems are the thinking process of mathematical theory research. Students research the problem can turn passive learning into active. They actively use the existing knowledge to explore, associate the unknown knowledge with the existing knowledge, which help students to build a better knowledge network.

\subsection{Example of Problems on Linear Representation of Vector}

Linear space is a core concept in linear algebra. The is the vital foundation in understand other concept in linear space. Its geometric intuition comes from two-dimensional plane and three-dimensional space, which can help student in understanding.

Problem3.1 Using geometric method to represent the following two-variable linear equations.

Solving and representing two-variables linear equations using geometric method is what students have known in middle school. Most students can easily make the image shown in Figure 1.

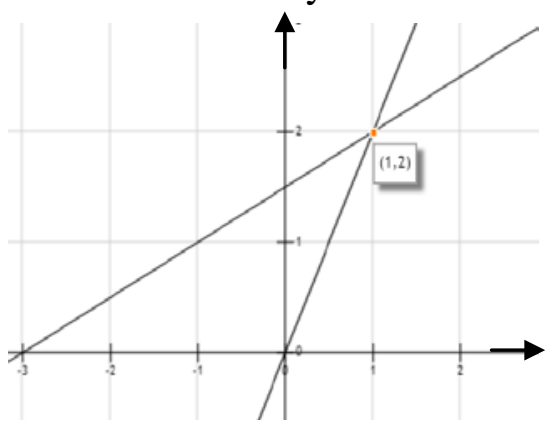

Figure 1: Line Graph of the Linear System.

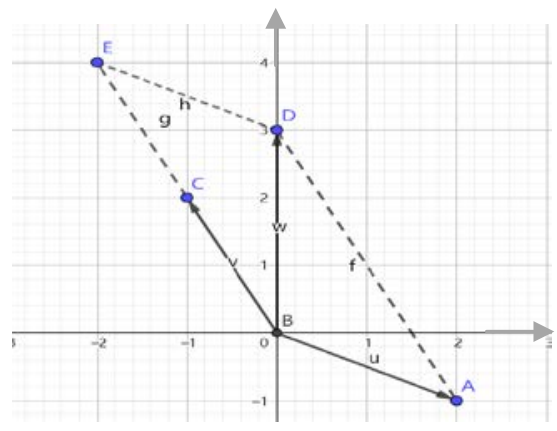

Figure 2:Vector Graph of the Linear System.

It can be found that the cross of the two lines is the solution of the system. Meanwhile, if we represent the system by vector form, it can be rewritten as

$$
\left(\begin{array}{c}
2 \\
-1
\end{array}\right) x_{1}+\left(\begin{array}{c}
-1 \\
2
\end{array}\right) x_{2}=\left(\begin{array}{l}
0 \\
3
\end{array}\right)
$$

which can also be represented by figure 2 . Since the above formula only include addiction and scalar multiplication which are linear operations, it can be claimed that vector $\left(\begin{array}{ll}0 & 3\end{array}\right)^{T}$ can be linear represented by vector $\left(\begin{array}{ll}2 & -1\end{array}\right)^{T}$ and vector $\left(\begin{array}{ll}-1 & 2\end{array}\right)^{T}$. Then students can generalized it to 
gain the general concept of linear representation. The study of this problem is helpful for students to understand the concept of linear representation from the perspective of geometric intuition, and also contribute for understanding the relationship between solving linear equations and vector space in the continues learning.

\subsection{Example of problems on general review}

In general engineering major, it usually set only 32 class hour for linear algebra course. The content of the course usually include determinant, matrix, vector and solving linear system. During the students' learning, they are hard to see the whole picture of the connections among different concepts. Therefore, in the period of general review in this course, teacher can guide students to summarize the relationships among different concepts and theorems, then construct a deeper knowledge network.

Problem4.1 What are the necessary and sufficient conditions forann-variable linear system $A x=b$ ( $A$ is a square matrix) to have unique solution?

This problem can help students to sort out the relationship among determinants, matrices, vectors, linear systems. Based on summarized, students can find several conditions. For instance, 1) $|A| \neq 0$;2) $R(A)=R(\bar{A})=n$; 3) $A$ is invertible; 4) $A$ is equivalent to identity matrix; 5) $b$ can be represented by the column vector of matrix Auniquely; 6 ) the row and column vector set of $A$ is linear independent. By research the inner relationship among different concepts, students can understand the concepts further so that they can apply them skillfully.

\section{Conclusions}

Problem-based learning based on the suitable problems, help student to learn the abstract concepts and theorems in linear algebra efficiently. It canenhance students' interest in learning and improve their innovation ability and application ability. The key of Problem-based learning is to design the problems suitable for the students' current cognition. This paper selects some contents in the course of linear algebra, design and analyze some problems can be used in practical teaching based on Problem based learning. The analysis and the examples are meaningful for both teaching theory and practice.

\section{Acknowledgements}

This work was supported by the Guangdong University of Petrochemical Technology under Grant Education and teaching reform research project(660685).

\section{References}

[1] Wu Xuejun, Tang Ming. (2008) Linear Algebra is Blue- The Course Design of <Linear Algebra> in Non-mathematic Major. University Mathematics, 2008(6): 12-16.

[2] Garding, L. (2012). Encounter with mathematics. Springer Science \& Business Media.

[3] Zhao Chunfang. (2019) Research on Linear Algebra Teaching Theory Based on Practical Application. Journal of Shandong Agricultural Engineering Institute. 2019, 36(02): 140-141.

[4] Fu Dongmei. (2014) Research and Implementation of Problem-basedLearning Method. University Education. 2014(4): 1-3.

[5] Long Bolin. (1985) Piaget's Constructive Learning Theory. Journal of Guizhou Normal College. 1985(1): 48-51.

[6] Zhu Lin. (2017) Teaching Research on the Concept of Linear Space Based on Generative Teaching Method. East China Normal University (Doctoral dissertation). 2017 
[7] Aydin, S. (2014). Using example generation to explore students' understanding of the concepts of linear dependence/independence in linear algebra. International Journal of Mathematical Education in Science and Technology, 45(6), 813-826.

[8] Wawro, M., Watson, K., \& Zandieh, M. (2019). Student understanding of linear combinations of eigenvectors. ZDM, 51(7), 1111-1123.

[9] Xiao Ying. (2016) Judgment and calculation method ofmatrix inverse. Advanced Mathematics Research. 2016.19(04):72-76. 